\title{
La création du dépôt de la Plaine de Baud à Rennes : les formes d'une stratégie spatiale, technique et architecturale (1909-1937)
}

The creation of the depot of the Plain of Baud in Rennes: the forms of a spatial, technical and architectural strategy (1909-1937)

\section{Christophe Le Bollan}

\section{OpenEdition Journals}

Édition électronique

URL : https://journals.openedition.org/rhcf/1819

DOI : 10.4000/rhcf.1819

\section{Éditeur}

Rails \& histoire

\section{Édition imprimée}

Date de publication : 1 décembre 2003

Pagination : 495-509

ISBN : 0996-9403

ISSN : 0996-9403

\section{Référence électronique}

Christophe Le Bollan, « La création du dépôt de la Plaine de Baud à Rennes : les formes d'une stratégie spatiale, technique et architecturale (1909-1937) », Revue d'histoire des chemins de fer [En ligne], 28-29 | 2003, mis en ligne le 05 janvier 2015, consulté le 22 avril 2022. URL : http://journals.openedition.org/ rhcf/1819; DOl : https://doi.org/10.4000/rhcf.1819 


\section{La création du dépôt de la Plaine de Baud à Rennes: les formes d'une stratégie spatiale, technique et architecturale (1909-1937)}

Le rachat, en 1909, de la Compagnie des chemins de fer de l'Ouest par l'État annonce pour les gares de la ligne Rennes-Brest un remaniement architectural important afin de satisfaire à de nouvelles exigences commerciales. Un effort particulier est ainsi réservé au service de la Traction, notamment à Rennes, où le dépôt, à l'efficacité limitée par son intégration aux ateliers de maintenance de la gare, réserve pour l'avenir des difficultés d'exploitation. Les Chemins de fer de l'État envisagent ainsi, dès 1911, son transfert sur un autre site, plus fonctionnel. Adopté dans le principe, ce projet révèle néanmoins des difficultés d'application, nées en particulier des exigences techniques imposées par les perfectionnements du service de la Traction. Il est en outre tributaire de l'extension de la gare de triage, à l'est du site, pour laquelle le processus d'expropriation s'avère plus complexe que prévu. Le projet définitif du dépôt n'est entériné qu'en 1930, après vingt années de réflexion durant lesquelles il va progressivement épouser les formes d'une stratégie spatiale, technique et architecturale.

\section{L'inadéquation du projet de 1911}

La gare de Rennes, dont l'emplacement est approuvé en 1855, occupe une position centrale dans la distribution du réseau breton. Cette disposition idéale favorise rapidement l'implantation d'installations techniques, en particulier celles de la Traction. En 1857, un dépôt important, formé de deux remises rectangulaires de $50 \mathrm{~m}$ de longueur placées de part et d'autre d'un pont tournant, est construit dans la partie sud de la gare (fig. 1). Puis, à partir de 1865, des ateliers de maintenance du matériel moteur et roulant sont aménagés dans cette même partie de la gare, englobant du même coup le dépôt, dont la capacité d'accueil passe à 56 machines par le regroupement en un seul bâtiment des deux remises d'origine. Formé en 1908 de 18 bâtiments, cet ensemble comprend un secteur ouest réservé aux voitures et un secteur est consacré aux locomotives. Malgré son caractère complémentaire, ce système présente quelques lacunes à l'arrivée des Chemins de fer de l'État, notamment pour la rationalisation des réparations, et le déplacement du dépôt devient dès lors inévitable. L’avant-projet du 30 octobre 1911 
désigne la boucle formée par la Vilaine, immédiatement à l'est du pont de Saint-Hélier, pour accueillir les nouvelles installations, la remise à machines initiale devant, quant à elle, être progressivement transformée en atelier de réparation (fig. 2). Estimé à 3250000 francs, ce dépôt comprend deux demi-rotondes d'une capacité de remisage de 72 machines sur voies rayonnantes, complétées par dix voies de réparations. Chaque demi-rotonde est desservie par un pont tournant de 23,50 m commandé électriquement. Un chantier à combustibles, d'une contenance de $20000 \mathrm{~m}^{3}$, est disposé entre les rotondes et les voies principales. La manutention du charbon est réalisée mécaniquement par une grue à commande électrique et avec des bennes à chargement automatique. Un atelier, des bureaux et un magasin sont insérés entre les deux demi-rotondes. La force motrice nécessaire pour la transmission principale, pour les machines-outils de l'atelier, pour le compresseur d'air, le tour à roues, et les motopompes de lavage de machines, est empruntée à l'usine électrique de la gare. Pour l'alimentation en eau, deux réservoirs de $700 \mathrm{~m}^{3}$ doivent être ajoutés aux deux réservoirs de $150 \mathrm{~m}^{3}$ existants, portant ainsi la réserve à $1000 \mathrm{~m}^{3}$, distribuée au moyen de neuf grues hydrauliques à grand débit. Enfin, le dépôt est complété par divers bâtiments : conciergerie, bains, lavabos, réfectoire et dortoirs pour le personnel roulant, maisons d'habitation pour le chef et les souschefs de dépôt ${ }^{1}$. Ce dessein se veut ambitieux mais sa réalisation est suspendue en raison de l'ouverture des hostilités en 1914. Durant le conflit, afin de soutenir l'effort de guerre des terrains sont acquis pour agrandir le triage de Saint-Hélier, mais aussi pour poser les jalons d'une nouvelle gare de triage sur la Plaine de Baud, vaste espace situé à l'Est, au-delà de l'embranchement de Châteaubriant. Cette nouvelle orientation compromet dans le même temps la boucle de la Vilaine, dont l'exiguiité, en définitive, est relevée par les ingénieurs.

\section{Le choix de la Plaine de Baud}

Sans surprise, au sortir de la guerre, le site de la Plaine de Baud est retenu pour accueillir le nouveau dépôt. Placé parallèlement, du côté sud, à la gare de triage projetée au même endroit, celui-ci, dont les plans sont adoptés en 1923, a l'avantage de bénéficier d'un large espace. Il reprend les dispositions de 1911 en les améliorant, avec dorénavant deux rotondes complètes, d'une capacité respective de 42 machines (rotonde est) et 46 machines (rotonde ouest), soit un total de 88 machines.

1- Lettre du ministre des Travaux publics au préfet d'Ille-et-Vilaine, 30 octobre 1911, Archives départementales d'Ille-et-Vilaine, 5 S 18. 


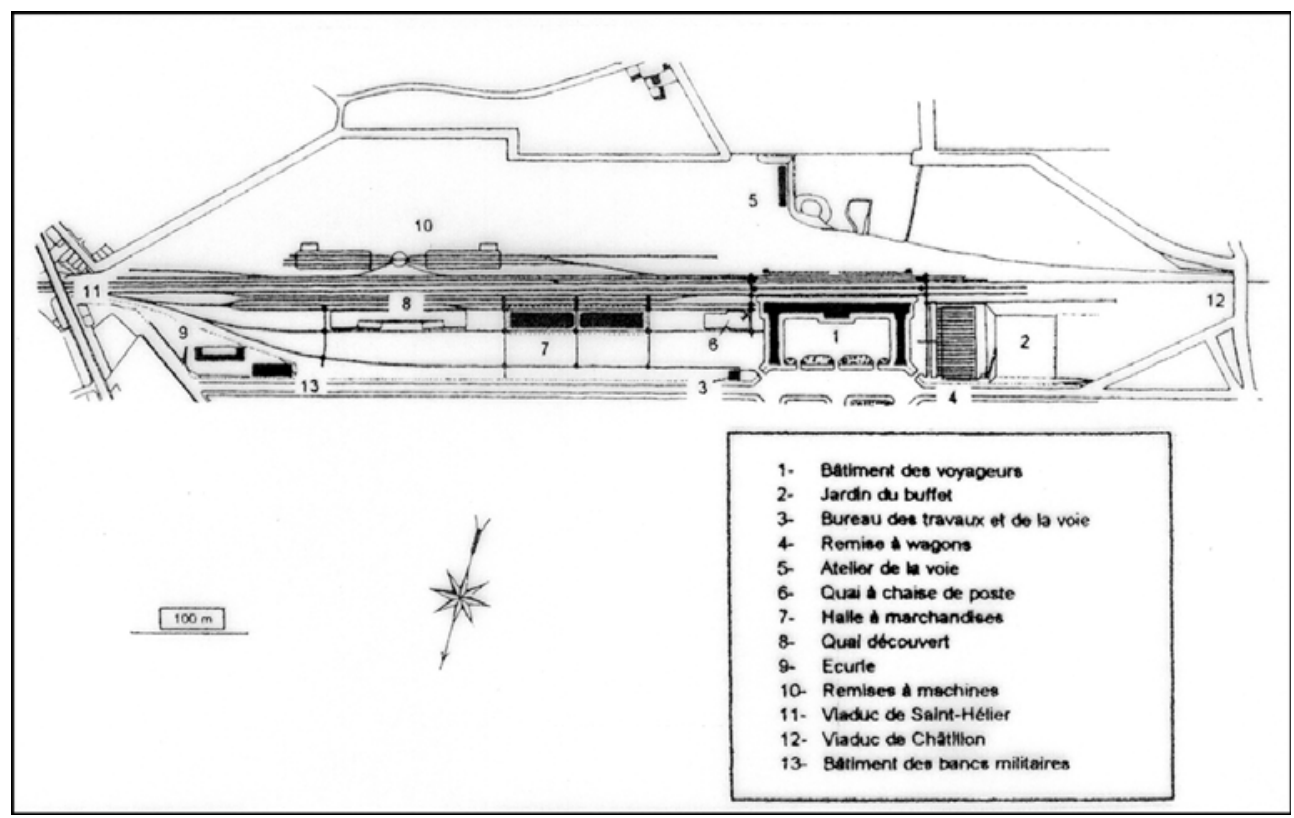

Figure 1. Plan de la gare de Rennes (1857). Plan C. Le Bollan.

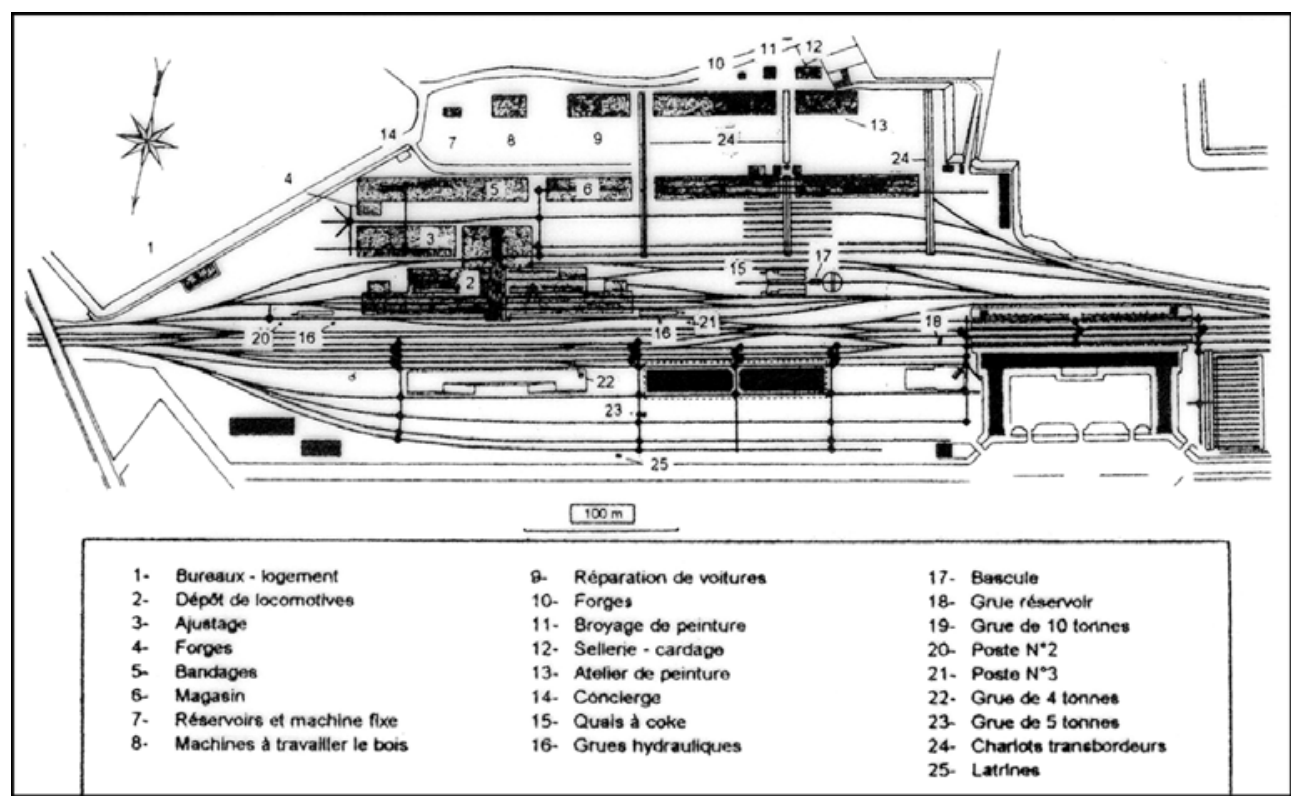

Figure 2. Plan de la gare de Rennes et des ateliers de maintenance du matériel moteur et roulant (1872). Plan C. Le Bollan. 
L'atelier de réparation initial inséré entre les rotondes, les maisons pour chef et sous-chefs de dépôt et les logements collectifs pour le personnel roulant sont conservés, tandis qu'un bâtiment pour lampisterie, atelier et désincrustants, ainsi qu'un magasin aux essences sont ajoutés au programme. Mais ce projet, pour lequel la compagnie fait le choix du béton armé pour la structure des bâtiments, est une nouvelle fois remis, probablement pour des raisons financières. Pendant ce temps, le dépôt de Saint-Hélier continue de fonctionner, malgré les désagréments de service.

L'arrivée de Raoul Dautry à la tête du réseau met un terme aux hésitations. Soucieux de faire avancer les différents chantiers engagés ou projetés dans de nombreuses gares, le nouveau directeur aspire avant tout à accélérer la modernisation de son entreprise, nécessaire pour en renforcer l'image de marque. À Rennes, cet objectif se concrétise notamment par la spécialisation des ateliers de maintenance, menée de 1930 à 1933³, tandis que, parallèlement, le site de la Plaine de Baud est enfin confirmé pour l'implantation du nouveau dépôt par la décision ministérielle du 23 juillet 1930, et de la nouvelle gare de triage par la décision ministérielle du 8 avril 19314. Inscrit dans la réorganisation du service des combustibles de la Traction, le dépôt comprend l'aménagement d'un parc de stockage de 65000 tonnes et d'un centre de mélange et de répartition des combustibles destinés à sa propre alimentation et à ses dix satellites (Brest, Châteaubriant, Dinan, Dol, Fougères, Saint-Malo, Morlaix, Saint-Brieuc, Laval et Segré). Au total, sa construction est évaluée à 25210000 francs comprenant, outre l'implantation de nouveaux bâtiments, l'établissement d'un faisceau de voies de service, d'un groupe de voies avec grues hydrauliques et fosses de visites, d'un gril de garage pour 36 machines, d'un triangle de tournage, d'une aire de lavage, et la fourniture d'énergie électrique pour l'éclairage

2- Raoul Dautry occupe ses fonctions de directeur des Chemins de fer de l'État du $1^{\text {er }}$ novembre 1928 au 11 juin 1937.

3- Les ateliers rennais, qui ont jusqu' alors réparé à la fois des locomotives, des voitures et des wagons, sont intégrés dans une politique générale de réorganisation et de spécialisation des grands ateliers du réseau. La priorité est accordée à l'augmentation du rendement et à l'amélioration de la qualité des réparations, tout en cherchant à réduire les frais d'exploitation. Ainsi, Rennes cesse de réparer des locomotives pour se consacrer à la révision des véhicules à moteur (draisines, locotracteurs, autorails), et se spécialise également dans la construction de wagons couverts.

4- La surface de terrains acquise pour l'extension du triage et l'implantation du dépôt se solde au total à 13 hectares. C'est de loin la plus grande extension réalisée à Rennes entre 1909 et 1937 (88,83\% des achats de terrains). 
et les divers moteurs ${ }^{5}$ (fig. 3 et 4). Quant au dépôt de Saint-Hélier, celuici est définitivement fermé en avril 1934, la remise à machines étant entièrement démantelée pour offrir de nouveaux espaces aux voies de desserte des ateliers.

\section{L'affirmation d'une homogénéité technique et archi- tecturale}

D’un point de vue architectural, ce nouveau dépôt, qui emploie près de 500 cheminots, est conçu pour fonctionner de manière autonome par rapport à l'ancienne configuration, avec des zones de travail et des zones de repos. Au total, le site comprend 14 bâtiments disposés suivant une logique technique. Alors que la Compagnie de l'Ouest avait privilégié l'emploi de matériaux traditionnels (pierre, brique, bois et métal), les Chemins de fer de l'État optent pour l'utilisation générale du béton armé pour l'ossature des constructions, tout en valorisant la recherche d'une homogénéité stylistique. Celle-ci se manifeste en particulier par le choix de toitures voûtées en berceau, employées pour six édifices.

Dans le domaine du remisage ${ }^{6}$, les deux rotondes suggérées en 1923 sont abandonnées au profit d'une remise rectangulaire dont les travaux sont confiés à la société parisienne Dumez (fig. 5) ${ }^{7}$.

5- Conseil de réseau, 29 décembre 1930, Archives nationales, Fonds Dautry, 307 AP 69, et 5 août 1932, Archives nationales, Fonds Dautry, 307 AP 70.

6- Sur le plan technique, les équipements intérieurs des remises bénéficient de quelques perfectionnements. Pour l'entretien des machines, le fusil, simple tube en acier alimenté à l'air comprimé ou à la vapeur, et raccordé à une prise fixe, est utilisé pour le ramonage des tubes à fumée. L'emploi de jets d'eau sous pression se généralise pour le lavage simple et rapide, à eau froide, des orifices de la chaudière des locomotives. D'autre part, la maitrise du remisage des machines, particulièrement les temps de stationnement, est améliorée. Pour les opérations de levage, les parcours kilométriques sont également mieux déterminés. Selon les séries et leurs qualités respectives, la périodicité kilométrique est variable : de $40000 \mathrm{~km}$ pour les machines de manœuvre à $120000 \mathrm{~km}$ et davantage pour les machines de vitesse. Le parcours entre deux levages étant pris comme unité, les locomotives subissent une visite périodique en pression tous les $1 / 16^{e}$ de parcours de levage et une visite périodique à froid en plus tous les $1 / 8^{\text {e }}$ de parcours de levage.

7- L'emploi de remises rectangulaires n'est pas systématique sur le réseau de l'État. Sur la ligne Rennes-Brest, par exemple, pour la construction du nouveau dépôt de SaintBrieuc à partir de 1922, il est fait usage d'une demi-rotonde à 28 voies rayonnantes, desservies par un pont tournant de 24 mètres de diamètre. À partir de 1937, huit voies seront réservées au remisage des autorails. 


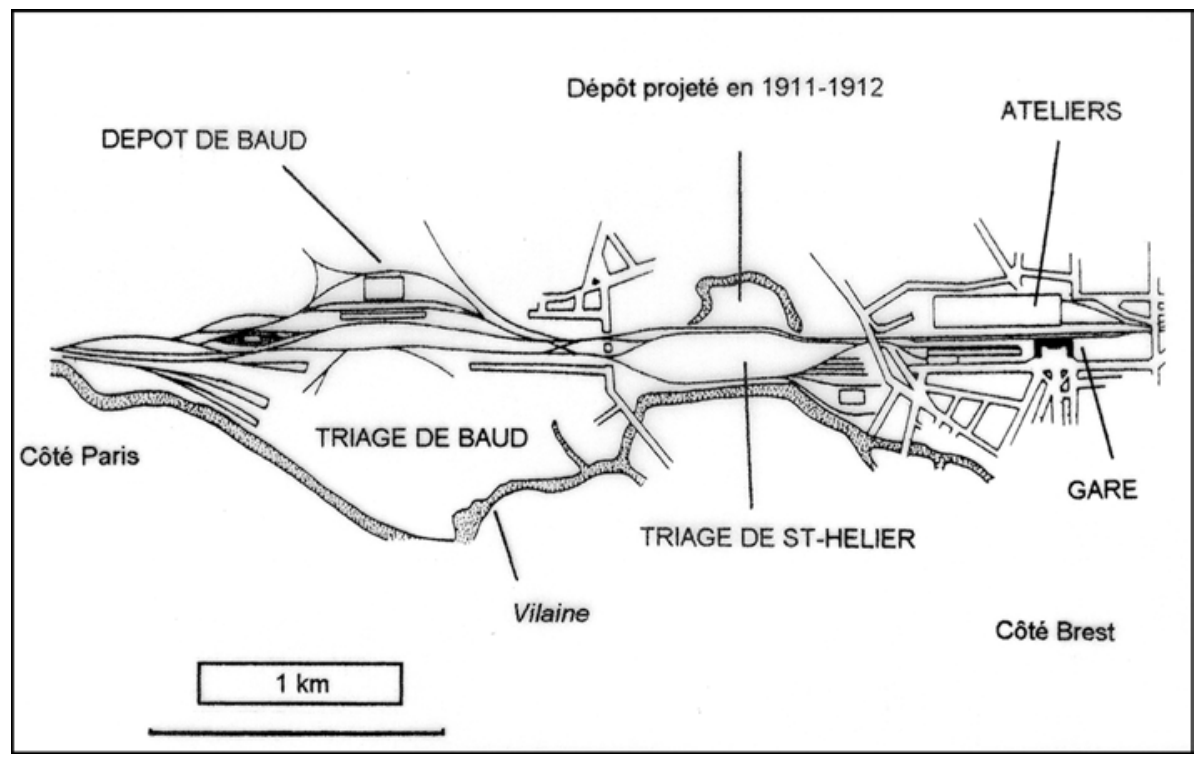

Figure 3. Disposition des installations ferroviaires de Rennes (1937). Plan C. Le Bollan.

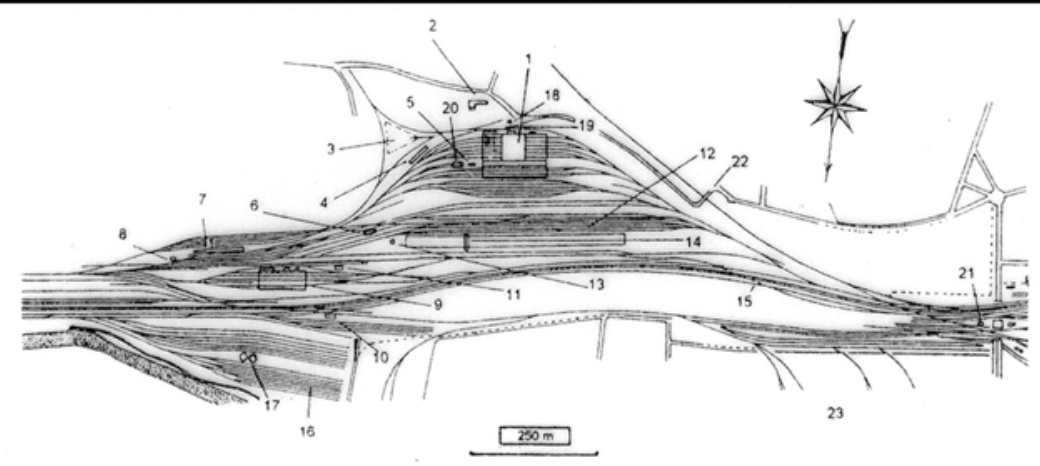

\begin{tabular}{|c|c|c|c|c|c|}
\hline 1. & Remise a machines & ๑. & Remise autorails & 17. & Reservoirs a eau \\
\hline 2. & Foyer des conducteurs & 10 & Poste $\mathrm{N}^{* 1}$ & 18. & Guertte du gardien \\
\hline 3. & Triangle de tournage & 11. & Huilerle, compresseur et parc a & 12 & Abri a bicyciettes \\
\hline 4 & Vestiaires ef cabinet medical & & sable & 20. & Aire de lavage \\
\hline 5. & Chaufterie & $12 \cdot$ & Parc a combustibies & 21. & Poste de traneformation \\
\hline 6. & Tobogoan & 13. & Ateller de la voile & 22- & Pont sut raik de ta rue Aluguate \\
\hline 7. & Babiment mouvement ef sabierie & 14 & Portique i combustibles & & Pavie \\
\hline 8. & $\begin{array}{l}\text { mecanique } \\
\text { Cave a easence }\end{array}$ & $\begin{array}{l}15 . \\
16 .\end{array}$ & $\begin{array}{l}\text { Saut de mouton } \\
\text { Voles de triage }\end{array}$ & 23. & Embr \\
\hline
\end{tabular}

Figure 4. Gare de Rennes, plan du dépôt et du triage de la Plaine de Baud (1937). Plan C. Le Bollan. 


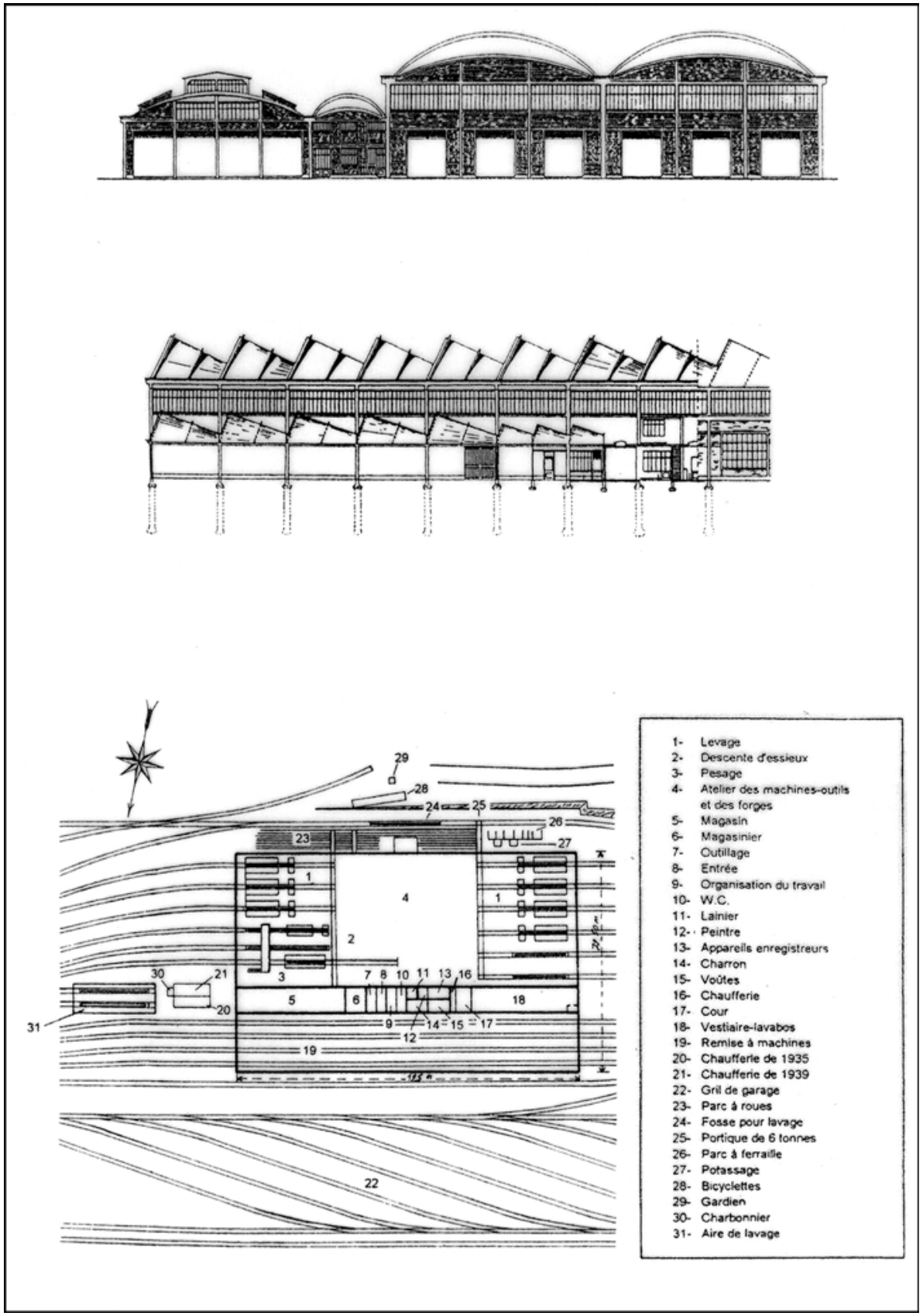

Figure 5. Remise à machines (façade ouest, coupe et plan, 1935), dépôt de la Plaine de Baud (Rennes). Direction régionale de la SNCF, Rennes, service de la Voie et des Bâtiments, cl. C. Le Bollan. 
Un compromis entre tradition et modernité, imprimé par l'emploi de la brique rouge pour les murs, permet des variétés de tons et de briser la monotonie endémique du béton. Sur le plan technique, le bâtiment, aux proportions importantes $(128 \mathrm{~m} \times 79,50 \mathrm{~m})$, a l'avantage de concentrer sur le même espace le remisage et les opérations d'ateliers, en trois parties principales. La première, la plus importante (environ $6400 \mathrm{~m}^{2}$ ), est située au Sud et abrite les ateliers des machines-outils, de levage des machines, de descente d'essieux (vérins) et de forges. La partie centrale est consacrée à divers locaux (lainier, magasin, magasinier, outillage, peintre, appareils enregistreurs, voûtes, organisation du travail, WC, charron, chaufferie, et vestiaire-lavabos), tandis que la troisième partie, au Nord, est réservée au remisage des machines. La toiture est formée longitudinalement de sheds pour les deux premières parties, qui diffusent un éclairage naturel important à l'intérieur des ateliers, la toiture de la remise étant, quant à elle, voûtée en berceau avec lanternon central. Une chaufferie supplémentaire en béton armé et briques est disposée au pignon est et se caractérise par sa silhouette élégante, avec toiture et lanternon central légèrement voûtés (fig. 6) ${ }^{8}$. Par ailleurs, l'alimentation en eau des machines est assurée par deux cuves en béton armé de $300 \mathrm{~m}^{3}$ posées sur piliers, situées à proximité de la Vilaine et desservant au total sept grues hydrauliques (une grue d'un débit minute de 2000 litres et six grues d'un débit minute de 5000 litres).

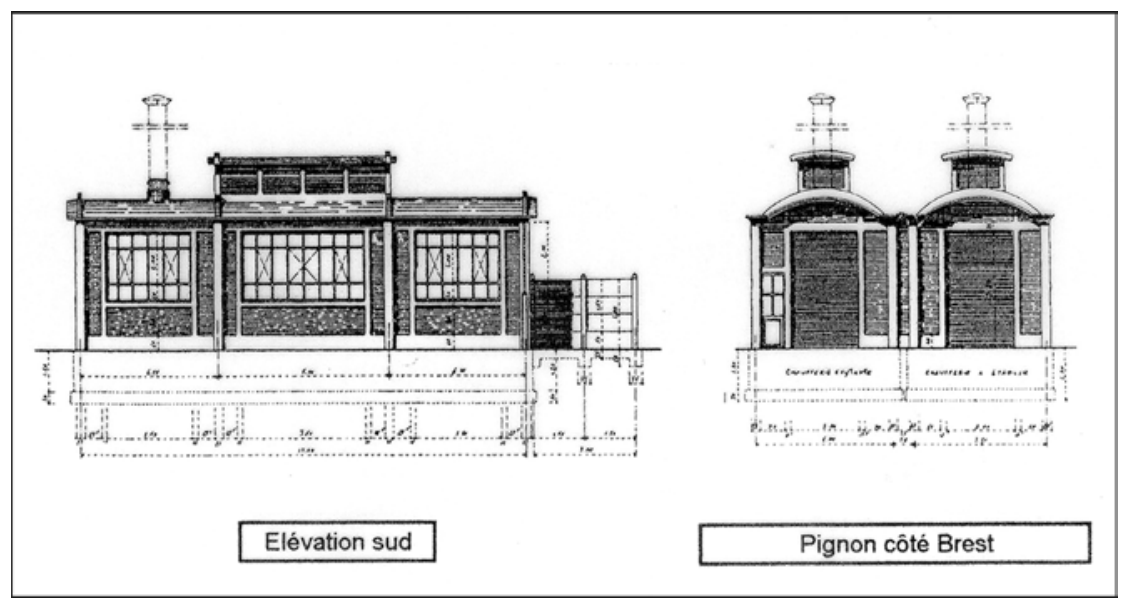

Figure 6. Chaufferie (disposition en 1939), dépôt de la Plaine de Baud (Rennes). Direction régionale de la SNCF, Rennes, service de la Voie et des Bâtiments, cl. C. Le Bollan.

8- Cette chaufferie sera doublée en 1939. 
Parallèlement, une remise à autorails (97 m x $12 \mathrm{~m}$ ), avec atelier de réparations, magasin et quatre fosses de visites (deux de $17 \mathrm{~m}$, une de 73,50 m et une de 68,50 m), est également construite à partir de 1935. L'année suivante, une chaufferie et une soute à mazout (d'une contenance de 15000 litres) sont accolées au bâtiment. La chaufferie assure en particulier la diffusion d'air chaud simultanément à l'intérieur de l'édifice (neuf aérothermes suspendus ou muraux), et à l'intérieur des autorails (trois aérothermes avec gaine). Suite à l'accentuation du transport par autorails dans la région rennaise, la remise est agrandie sur le côté nord à partir de 1937. La partie rajoutée (ateliers de réparations) accueille trois nouvelles voies avec fosses de visite : deux voies type « autorails » Z a 19 (90 m et $35 \mathrm{~m}$ ) et une voie type «vapeur» Z a 13 (65 m). En élévation, les ateliers (19 travées de hautes fenêtres) sont plus élevés que la remise initiale. Le toit, scandé de nervures transversales et achevé d'un lanternon, reprend la forme en berceau de la remise d'origine (fig. 7).

Dans le domaine de la distribution des combustibles, le dépôt est pourvu de quatre bâtiments (sablerie mécanique, toboggan, cave à essence, et bâtiment pour compresseur, huilerie et parc à sable). La sablerie mécanique $(63,80 \mathrm{~m}$ x 5,70 m) comprend un parc à sable, un local à désincrustant, un quai haut, une huilerie, une lampisterie, un vestiaire-lavabos, un bureau d'affichage, un bureau pour sous-chefs, un local pour téléphone et graphique, un magasin annexe et un atelier. Le système de manutention mécanique, installé dans une tour, permet d'assécher progressivement le sable par le jeu d'un élévateur vertical. Le sable est ainsi acheminé vers un foyer et un sécheur, puis dirigé vers un portique de $50 \mathrm{~m}$ de portée, lequel, desservi par quatre voies, alimente les tenders des locomotives par un système de goulottes de versement (fig. 8). Parallèlement, pour la distribution du charbon, le toboggan, complété à proximité par un portique pour chantier à combustibles, est doté d'un système identique de manutention. Techniquement, les wagons, dont le mouvement doit être régulier pour fiabiliser l'efficacité du système, sont déchargés, par simple gravité, dans des trémies enterrées. Le charbon est ensuite dirigé vers les trémies de distribution par une chaîne à godets et des tapis roulants installés dans un silo-portique, puis également distribué aux locomotives par des goulottes de distribution (fig. 4, $\mathrm{n}^{\circ}$ 14). La cave à essence, dont la parfaite étanchéité est requise lors de sa construction, est semi-enterrée et accessible par une rampe et deux grands vantaux en tôle. L'éclairage indirect et la nécessaire aération sont assurés par une série de petits châssis métalliques disposée dans la partie supérieure de l'élévation (fig. 9). 


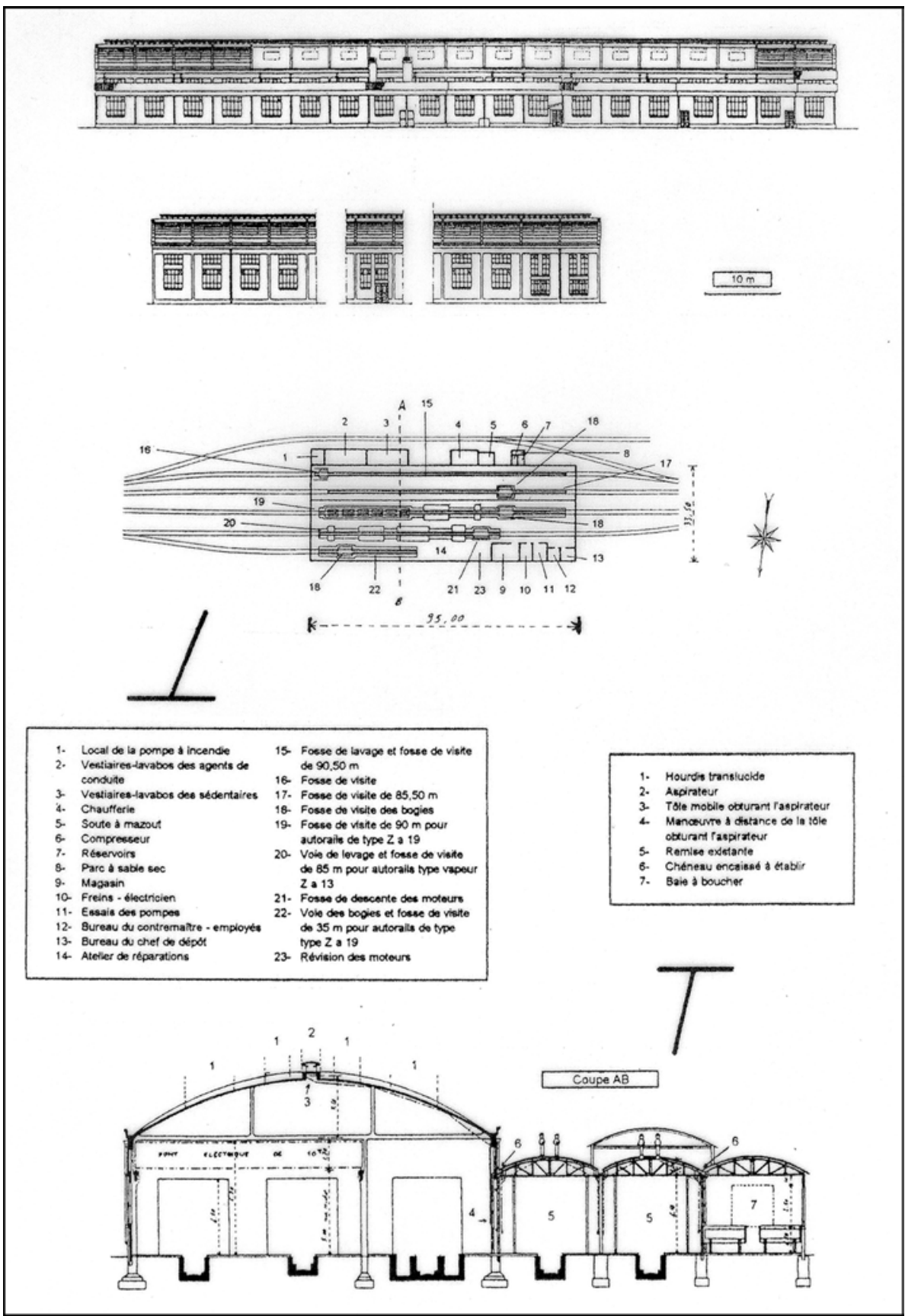

Figure 7. Remise à autorails (façades sud et nord, plan et coupe, 1937), dépôt de la Plaine de Baud (Rennes). Direction régionale de la SNCF, Rennes, service de la Voie et des Bâtiments, cl. C. Le Bollan. 


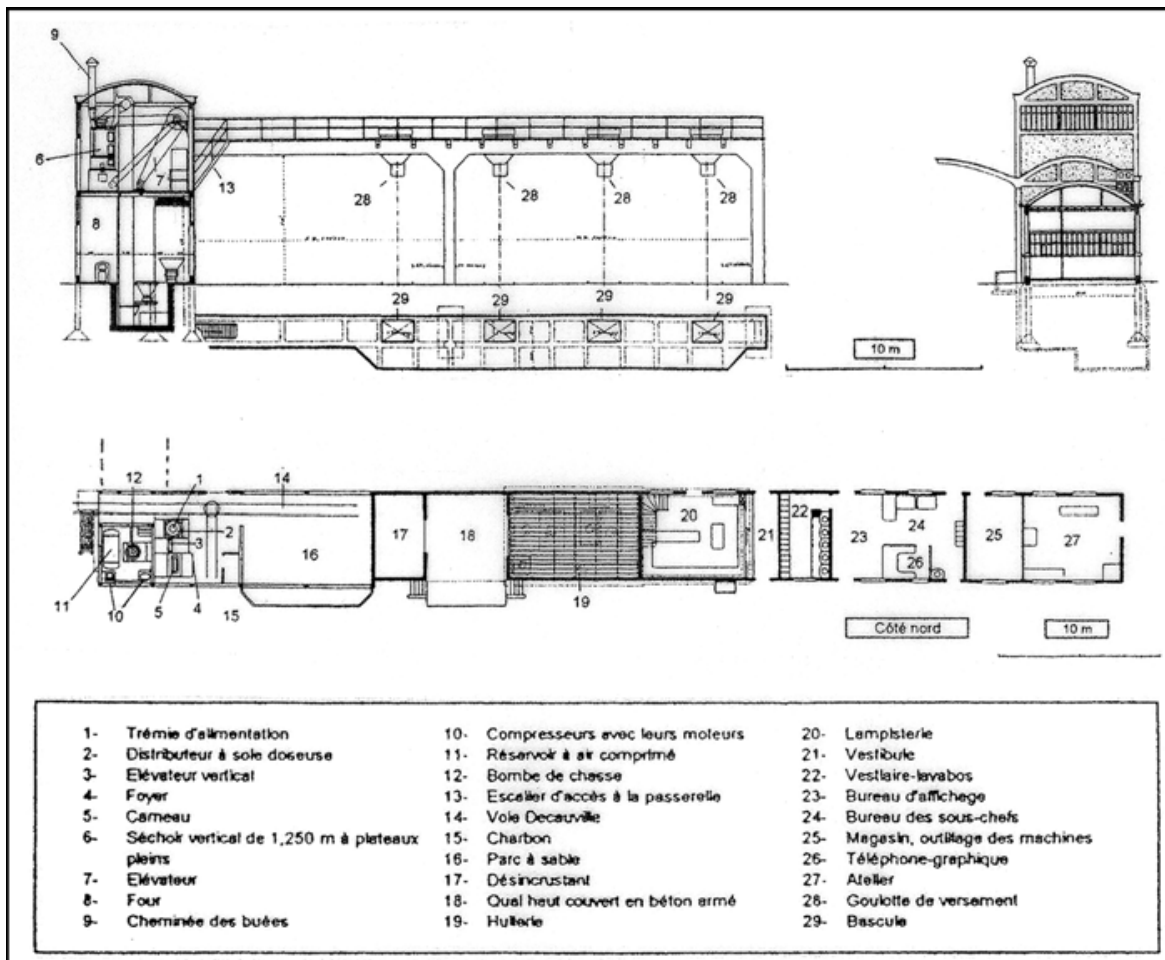

Figure 8. Bâtiment "mouvement " et sablerie mécanique (élévations diverses et plan, 1934), dépôt de la Plaine de Baud (Rennes). Direction régionale de la SNCF, Rennes, service de la Voie et des Bâtiments, cl. C. Le Bollan.

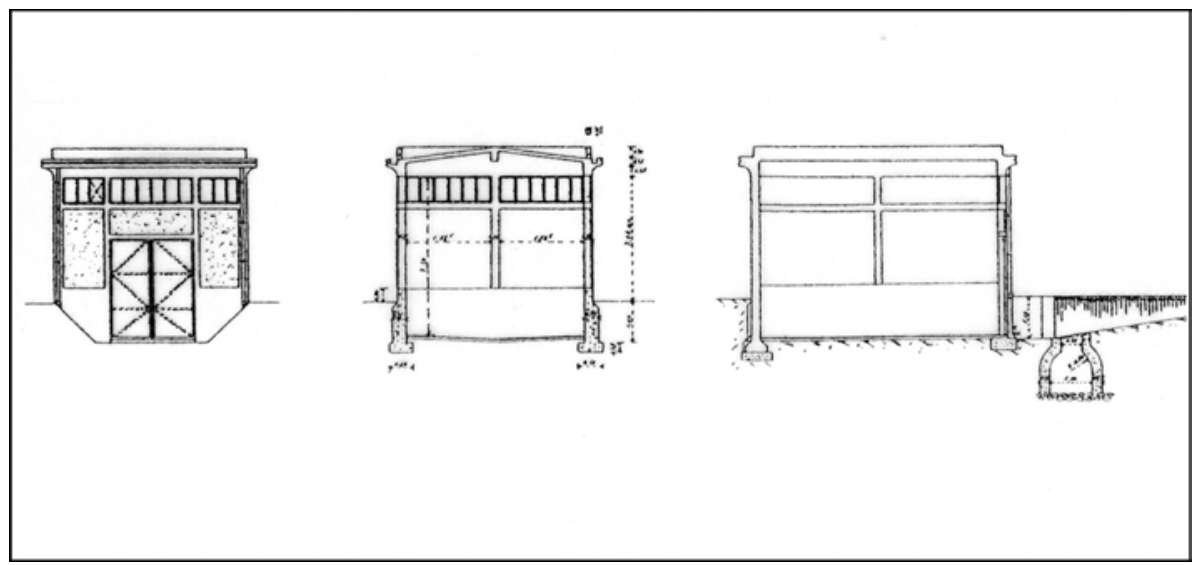

Figure 9. Cave à essences (façade ouest et coupes diverses, 1935), dépôt de la Plaine de Baud (Rennes). Direction régionale de la SNCF, Rennes, service de la Voie et des Bâtiments, cl. C. Le Bollan. 
Le bâtiment pour compresseur, huilerie et parc à sable (8,50 m x 6,30 m) comprend trois locaux (compresseur, dépôt de sable sec, et huilerie). Un dispositif pour l'emmagasinage du sable est posé à l'extérieur (fig. 10) tandis qu'un réservoir métallique de $6 \mathrm{~m}^{3}$ est aménagé à proximité du bâtiment pour recueillir les huiles usagées.

L'efficacité du dépôt est enfin renforcée par une guérite pour le gardien, un abri à bicyclettes, un atelier de la Voie, un bâtiment pour vestiaires (agents de conduite) et cabinet médical et un foyer pour le personnel roulant. Les deux premières constructions sont élaborées en matériaux légers (brique et bois). L'atelier (7,50 m x 4,50 m), d'une architecture simple avec toiture à deux pans en ardoises, abrite deux pièces (atelier et magasin) accessibles par les pignons (fig. 11). Le bâtiment pour vestiaires, implanté à l'est de la remise à machines, présente un aspect allongé $(52,38 \mathrm{~m} \times 8,84 \mathrm{~m})$ avec toiture légèrement voûtée (fig. 12). Le foyer, quant à lui, se caractérise par sa silhouette de nature urbaine, spécifiée par une toiture classique à deux pans en ardoises, une incrustation de pierres de taille, et un plan en L. Propice au repos, il offre une capacité d'accueil de vingt-deux chambres, disposées sur trois niveaux, avec lavabos, W.C. et réfectoire (fig. 13).
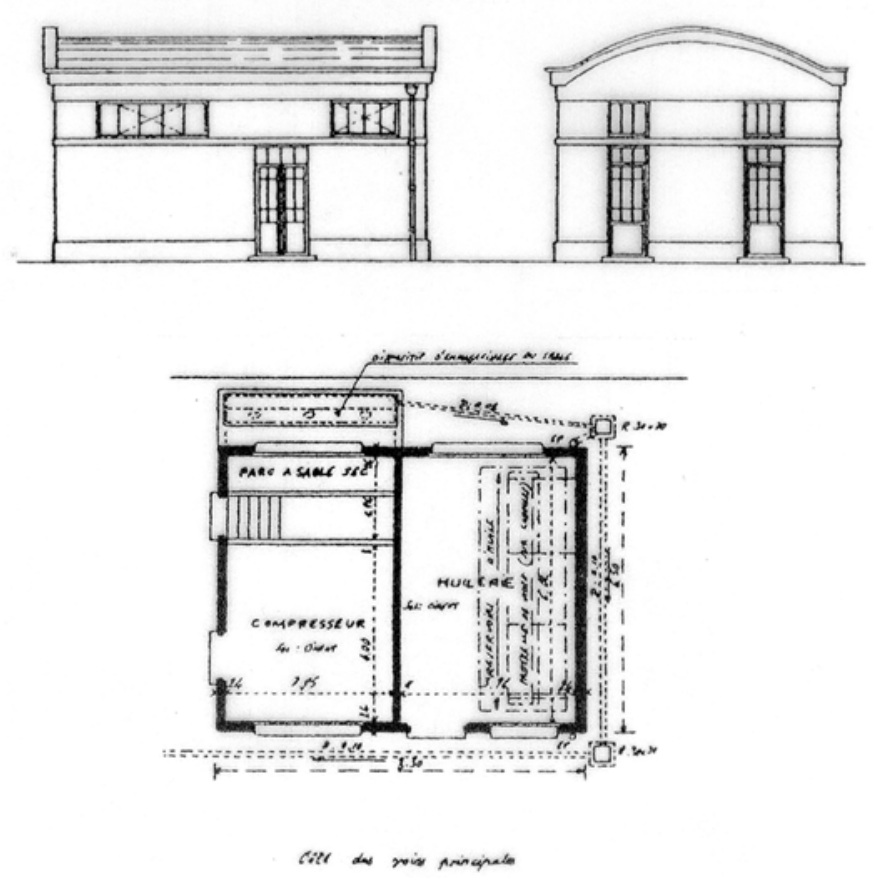

Figure 10. Bâtiment pour compresseur, huilerie et parc à sable (élévations diverses et plan, 1935), dépôt de la Plaine de Baud (Rennes). Direction régionale de la SNCF, Rennes, service de la Voie et des Bâtiments, cl. C. Le Bollan. 

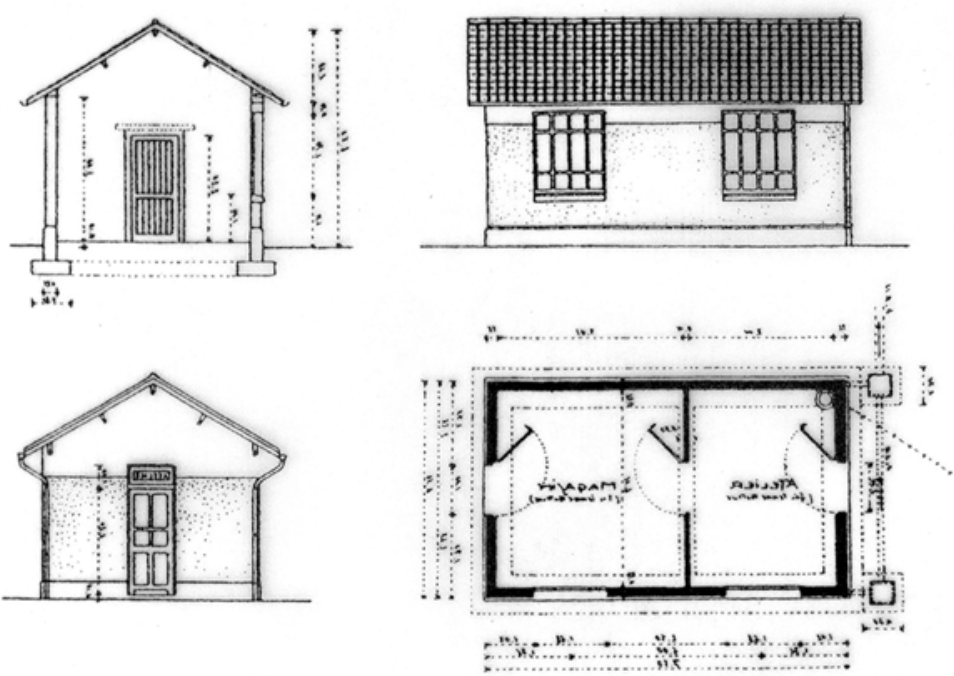

Figure 11. Atelier de la voie (élévations diverses et plan, 1935), dépôt de la Plaine de Baud (Rennes). Direction régionale de la SNCF, Rennes, service de la Voie et des Bâtiments, cl. C. Le Bollan.

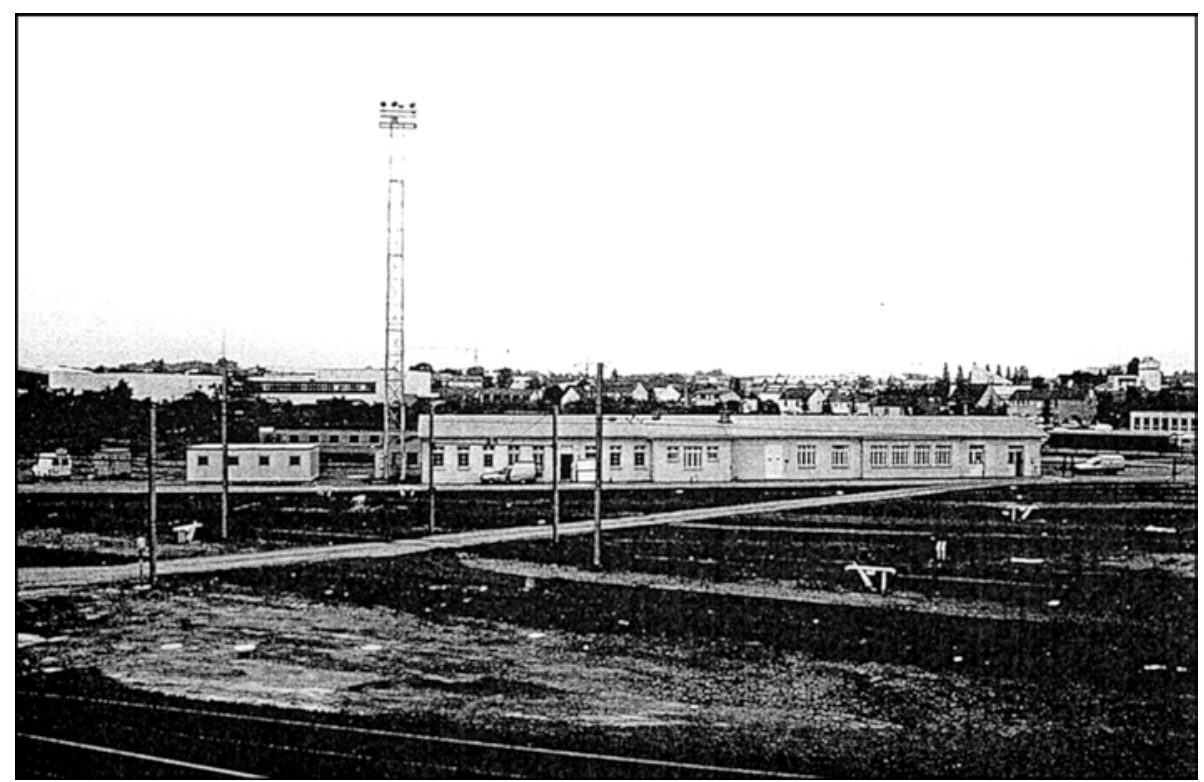

Figure 12. Bâtiment des vestiaires et du cabinet médical (vue actuelle nord-ouest), dépôt de la Plaine de Baud (Rennes). Cl. C. Le Bollan. 


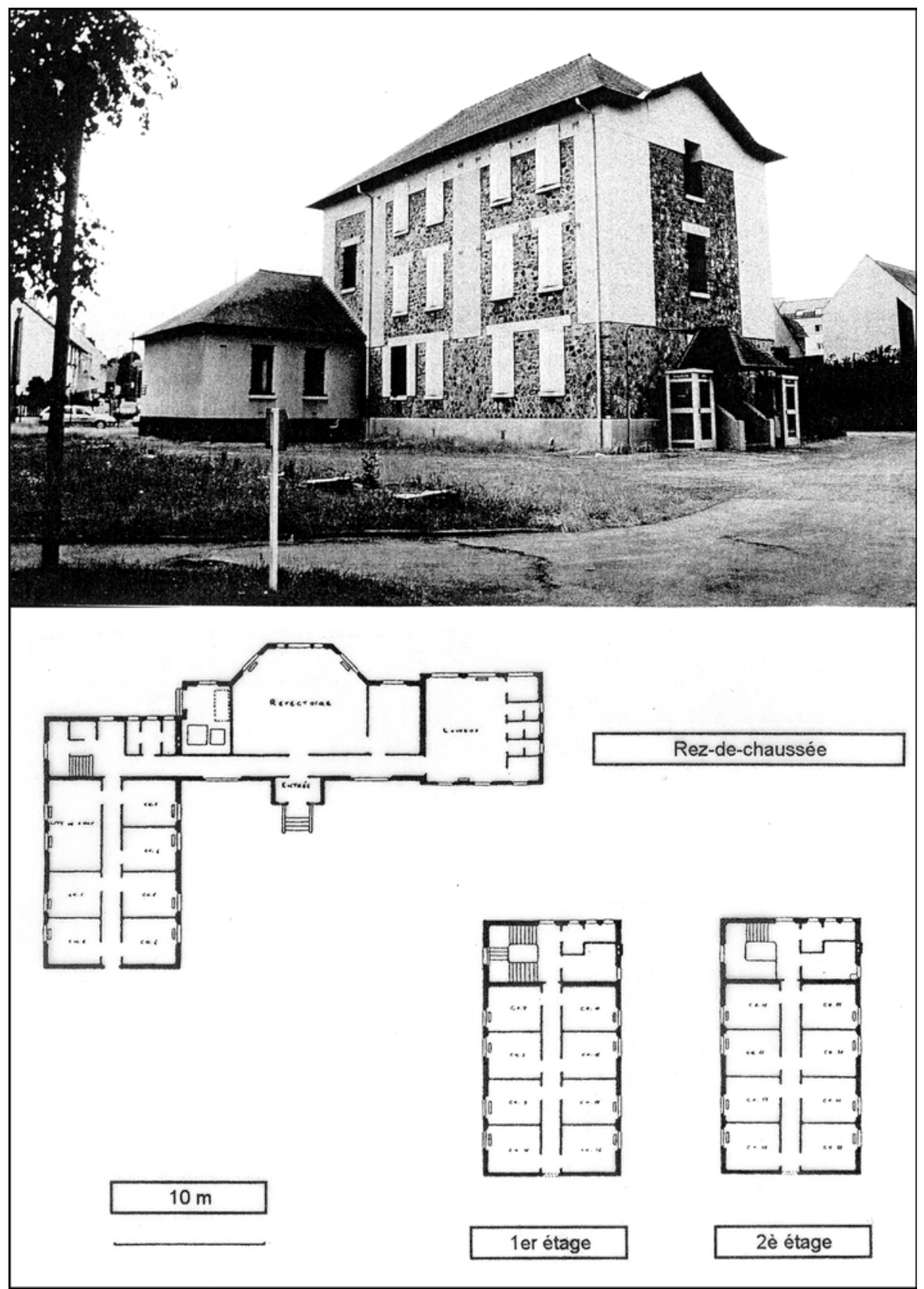

Figure 13. Foyer du personnel roulant (vue actuelle nord-est et plans divers de 1935), dépôt de la Plaine de Baud (Rennes). Cl. C. Le Bollan et documents de la direction régionale de la SNCF, Rennes, service de la Voie et des Bâtiments. 
Finalement, la construction du dépôt de la Plaine de Baud, par sa cohérence technique, contribue au rééquilibre spatial des installations ferroviaires rennaises. Elle permet en outre l'application d'un programme architectural d'envergure qui, par son approche pragmatique, amplifie la conscience d'un réseau d'État aménageur du territoire. Entre propagande technique et obligation de s'adapter aux nouveaux procédés de construction, ce dépôt renforce également le rôle régional de la gare de Rennes. Par la suite, au temps de la SNCF, le site va progressivement s'harmoniser avec l'électrification des lignes de l'Ouest. Plusieurs bâtiments et équipements construits entre 1934 et 1937 vont ainsi disparaittre par nécessité de service, à l'exception de la remise à machines, du bâtiment des vestiaires et du foyer des conducteurs, seuls témoins du temps révolu de la vapeur. 